\title{
Evaluation of Cellular and Systemic Toxicity of Dielectric Barrier Discharge Plasma-Treated $N$-Acetylcysteine as Potential Antimicrobial Catheter Lock Solution
}

\author{
Utku K. Ercan $^{1}$ (D), Adam D. Yost ${ }^{2,3}$, Kimberly Wasko ${ }^{3}$, Ari D. Brooks ${ }^{3}$ and Suresh G. Joshi ${ }^{2,3, *(D)}$ \\ 1 Department of Biomedical Engineering, İzmir Katip Çelebi University, Çiğli, 35620 İzmir, Turkey; \\ utkuk.ercan@ikc.edu.tr \\ 2 School of Biomedical Engineering, Science and Health Systems, Drexel University, \\ Philadelphia, PA 19104, USA; adam.d.yost@gmail.com \\ 3 Center for Surgical Infections, Department of Surgery, College of Medicine, Drexel University, \\ Philadelphia, PA 19102, USA; kaworganic@gmail.com (K.W.); ari.brooks@uphs.upenn.edu (A.D.B.) \\ * Correspondence: sgj24@drexel.edu
}

Citation: Ercan, U.K.; Yost, A.D.; Wasko, K.; Brooks, A.D.; Joshi, S.G. Evaluation of Cellular and Systemic Toxicity of Dielectric Barrier Discharge Plasma-Treated

$N$-Acetylcysteine as Potential Antimicrobial Catheter Lock Solution. Plasma 2021, 4, 732-744. https:// doi.org/10.3390/plasma4040036

Academic Editors:

Katharina Stapelmann, Savino Longo and Pietro Ranieri

Received: 6 August 2021

Accepted: 18 October 2021

Published: 26 October 2021

Publisher's Note: MDPI stays neutral with regard to jurisdictional claims in published maps and institutional affiliations.

Copyright: (c) 2021 by the authors. Licensee MDPI, Basel, Switzerland. This article is an open access article distributed under the terms and conditions of the Creative Commons Attribution (CC BY) license (https:/ / creativecommons.org/licenses/by/ $4.0 /)$.

\begin{abstract}
Intravenous catheter-related bloodstream infections are a cause of remarkable problems. Catheter lock solutions are used to keep catheter patency and prevent catheter-related bloodstream infections. The leakage of catheter lock solution to the bloodstream raises toxicity concerns. Plasmatreated liquids carry the potential to act as catheter lock solutions by virtue of their strong antimicrobial effects. The present study investigates the tolerance of the proposed solution in vitro and in vivo. $\mathrm{N}$-acetylcysteine (NAC) solution was treated with atmospheric-air DBD plasma and antimicrobial assays were performed. The cytotoxicity of the plasma-treated NAC solution was evaluated on an EA.hy926 cell line. Intravenous administration of plasma-treated NAC solution in different doses was given to Sprague Dawley rats. One week after infusion with plasma-treated NAC solution, first, the blood samples were collected, and then liver, kidney, tail vein, heart, and lung tissue samples were collected from euthanized rats for histopathological examination. The cytotoxicity of plasmatreated NAC solution depended on plasma treatment time, contact time, and cell number. A strong antimicrobial effect with no cytotoxicity of plasma-treated NAC solution was observed in endothelial cells. Based on blood tests and histopathological examination, no signs of systemic toxicity were observed that can be correlated to the plasma-treated-NAC solution. This solution has the potential to be used as a catheter lock solution with strong antimicrobial properties, keeping catheter patency.
\end{abstract}

Keywords: acute systemic toxicity; antimicrobial; bloodstream infection; catheter-related biofilm; cold plasma; dielectric barrier discharge; lock solution; plasma medicine

\section{Introduction}

Access to the vascular system is one of the most essential practices of modern medicine especially for hospitalized patients and $70 \%$ of them receive some type of catheter for vascular access $[1,2]$. Intravascular catheters are devices that are used for vascular entry and comprise various devices such as ports, peripheral and midline venous catheters, central venous catheters (CVCs), and arterial catheters [2]. These devices serve several purposes including monitoring hemodynamic parameters of the patient, blood collection for diagnostic or prognostic tests, administration of intravenous fluids and medications, and linking patients to hemodialysis machines [3]. Catheters are foreign bodies, remain in contact with skin and soft tissues, and serve as the perfect surface for bacterial biofilm formation that may subsequently cause bloodstream infections (BSIs) [2,4]. Catheter-related bloodstream infection (CRBSI) and central line-associated bloodstream infection (CALBSI) are two different BSIs that are related to intravascular catheters [3,5]. CRBSI is, more likely a clinical expression that requires microbiological methods to directly identify the catheter as the source of BSI. However, CLABSI refers to the presence of BSI in patients who have 
central venous catheters [2,3]. The presence of a catheter plays a key role in the pathogenesis of BSIs as it serves as a suitable surface for clotting and subsequent microbial adhesion and biofilm formation [6,7]. CLABSIs have a mortality rate of around $12 \%$, adds about 7 days of hospitalization, and bring approximately USD 45,000 of economic burden per patient to the healthcare system in the United States of America alone, and makes them one of the costly nosocomial infections [8,9]. Eradication of biofilms that are formed in catheter ports is crucial to overcome CLABSI [6]. However, biofilms exhibit higher resistance against various antimicrobial agents including antibiotics compared to planktonic forms of pathogens [10]. This increased resistance is primarily due to limited penetration of antimicrobials to biofilmembedded pathogens by the presence of an extracellular polymeric substance (EPS) that is secreted by pathogens during the formation of biofilm [11].

Despite the reduction of the CLABSI by the practice of fundamental infection control methods, further methods are needed for prevention and control of it [12]. Catheter lock solutions (antimicrobial lock solutions) along with catheter flushing have been shown to reduce CLABSI and CRBSI [12,13]. Cleaning of the catheter lumen by injection of $0.9 \% \mathrm{NaCl}$ (normal saline) solution is defined as catheter flushing. Flushing is followed by catheter locking that involves the injection of catheter lock solution (a solution that has antimicrobial activity) in a volume that could fill the lumen of the catheter when the catheter is not in use to prevent the clot formation and inhibit microbial colonization, and biofilm formation [13]. The lock solution was first practiced in 1988 for the treatment of catheter-related sepsis in parenteral nutrition patients, involved locking of the catheter by antibiotics $[13,14]$. The antimicrobial lock therapy requires the injection of concentrated antimicrobial agents that are 100-1000 times higher than the minimum inhibitory concentration (MIC) due to the increased resistance of biofilms to antimicrobials. Antimicrobial lock solutions are classified as antibiotic and non-antibiotic lock solutions which involve antimicrobials that are not indicated for systemic use [8]. Catheter lock solutions are composed of usually an anticoagulant substance such as heparin and concentrated antimicrobial agent [15]. However, currently used catheter lock solutions have some drawbacks such as possible promotion of biofilm formation by heparin [16]. The antibiotic lock solutions may lead to the development of antibiotic resistance, leakage of lock solution to blood-stream may cause systemic toxicity and allergic reactions $[4,13]$. Therefore, toxicity evaluation of catheter lock solution is advisable.

Over the past two decades, non-thermal atmospheric dielectric-barrier discharge (DBD) plasma is increasingly employed for surface-associated decontamination [17]. Recently, our laboratory has demonstrated that liquids activated with non-thermal plasma acquire strong antimicrobial properties, and these generated solutions retain antimicrobial properties at room temperature for extended periods of time [18]. These solutions efficiently inactivate both the planktonic and biofilm forms of pathogens including multi-drug resistant (MDR) organisms [18,19]. Our investigations also revealed that the antimicrobial property of the solutions is contributed by reactive oxygen species (ROS) and reactive nitrogen species (RNS) that are formed by various molecular mechanisms of reactive species and the interactions of products therein [20]. A range of products are generated from nonthermal plasma treatment of various liquids including water, saline solution phosphatebuffered saline solution, which exhibits broad-spectrum antimicrobial property $[18,21]$, and anti-biofilm property against MDR pathogen biofilms [18]. Previously, our group has reported the broad-spectrum antimicrobial effect of plasma-treated $N$-acetylcysteine (NAC) solution on biofilms of Gram-positive, Gram-negative, and fungal pathogens that were grown on polyurethane catheters [18]. Despite the NAC being an antioxidant agent, it is chemically modified by non-thermal atmospheric plasma treatment and converted to a potent antimicrobial agent and a source of ROS and RNS that inactivate pathogens via oxidative and nitrosative damage [20,22]. Therefore, we decided to investigate further on plasma-treated NAC solution and its systemic effect on vital organs in animals (rats), so a possibility of skin and tissue associated applications can be explored; such as potential catheter lock solution to combat against CRBSI and CLABSI. 
The aim of the present study is to evaluate antimicrobial efficacy in the presence of blood, the short-term cytotoxicity and acute systemic toxicity of plasma-treated NAC solution, using endothelial cell culturing (in vitro), and the systemic toxicity by intravenous administration in rats (in vivo).

\section{Materials and Methods}

\subsection{Plasma Source and Plasma Treatment of NAC Solution}

In the present study, $5 \mathrm{mM}$ NAC solution was treated with DBD atmospheric (ambient air) plasma (non-thermal plasma). The NAC powder was weighed and dissolved in $1 \mathrm{X}$ sterile PBS solution (both, Sigma Chemical Co., St. Louis, MO, USA) and filter sterilized to obtain $100 \mathrm{mM}$ stocks of NAC that were aliquoted and stored at $-20{ }^{\circ} \mathrm{C}$. Stocks of NAC solutions were thawed and diluted to $5 \mathrm{mM}$ of working concentration in 1X sterile PBS solution before each experiment. NAC solution was treated with DBD air plasma that was generated by a microsecond pulsed AC power supply, operated at $31.4 \mathrm{kV}$ peak to peak voltage and $1.5 \mathrm{kHz}$ frequency and $0.29 \mathrm{~W} / \mathrm{cm}^{2}$ power distribution. NAC solution was treated for either 1,2 , and 3 min separately at $2 \mathrm{~mm}$ of discharge gap or left untreated (0 min). The plasma set-up was as described previously [18,20,22].

\subsection{Cytotoxicity of Plasma-Treated NAC Solution}

Cytotoxicity of plasma-treated NAC solution was tested on the EA.hy926 human umbilical vein endothelial cell line. Frozen stocks of cell line were thawed in water bath at $37^{\circ} \mathrm{C}$ for $3 \mathrm{~min} .1 \mathrm{~mL}$ of thawed cell aliquot was transferred to $15 \mathrm{~mL}$ centrifuge tube and diluted with 1\% FBS (Fetal Bovine Serum; Sigma) containing 9 mL of DMEM (Dulbecco's Modified Eagle Medium) (Invitrogen, Grand Island, NY, USA). Afterward, cells were centrifuged at $1500 \mathrm{rpm}$ for $5 \mathrm{~min}$ and the supernatant was removed. Ten milliliters of fresh DMEM (containing 10\% FBS and 1\% penicillin-streptomycin (Sigma) solution) was added to the cell pellet and cells were gently homogenized by pipetting and transferred into a T25 cell culture flask (Corning, Corning, NY, USA), and incubated in $\mathrm{CO}_{2}$ incubator at $37{ }^{\circ} \mathrm{C}$ for $48 \mathrm{~h}$ until cells became $90 \%$ confluent. Confluent cells were trypsinized and counted with a hemocytometer to calculate the approximate cell number to $\sim 20,000$ to seed in a 96 well-plate. Ten percent FBS and 1\% penicillin-streptomycin containing $200 \mu \mathrm{L}$ of fresh DMEM was added to cells in each well and cells were incubated in a $\mathrm{CO}_{2}$ incubator at $37{ }^{\circ} \mathrm{C}$ until they get $90 \%$ confluent ( 30,000 cells/well). Confluent cells were washed with $1 \mathrm{X}$ sterile PBS twice and $100 \mu \mathrm{L}$ of serum-free DMEM was added to cells. Then, $100 \mu \mathrm{L}$ of untreated and 1,2, and 3-min plasma-treated NAC solutions were added to the cells and held for 5, 10, 15, and $30 \mathrm{~min}$. After exposure of cells to plasma-treated NAC solution, media and plasma-treated NAC solution were removed; cells were washed with 1X sterile PBS twice and 10\% FBS and 1\% penicillin-streptomycin containing $200 \mu \mathrm{L}$ of fresh DMEM was added. Cells were incubated in a $\mathrm{CO}_{2}$ incubator at $37{ }^{\circ} \mathrm{C}$ for 24 and $48 \mathrm{~h}$ for recovery. Serum-free DMEM and $4 \%$ chlorhexidine (Sigma-Aldrich, Inc., St. Louis, $\mathrm{MO}, \mathrm{USA}$ ) solution were used as negative and positive controls, respectively. Following the recovery period, cell viability was determined by XTT (2,3-Bis-(2-Methoxy-4-Nitro-5Sulfophenyl) (Sigma-Aldrich) assay. Prior to the XTT assay, cells were washed with $1 \mathrm{X}$ sterile PBS once and $200 \mu \mathrm{L}$ of $0.5 \mathrm{mg} / \mathrm{mL}$ XTT solution was added to cells and incubated for $4 \mathrm{~h}$ at $37^{\circ} \mathrm{C}$. Then $150 \mu \mathrm{L}$ of reacted XTT solution was gently collected from each well and transferred to a new well and the absorbance read at $496 \mathrm{~nm}$. A set of cells that were exposed to serum-free media were included as a positive control group. The viability of the control group for each plasma treatment time, exposure time, and recovery time were set to $100 \%$, and the percentage of viable cells that were exposed to plasma-treated NAC solution normalized according to the positive control group. Each experiment was carried out a minimum of three times in triplicate. 


\subsection{Effect of Blood on Antimicrobial Effect of Plasma-Treated NAC Solution}

The antimicrobial activity of plasma-treated NAC solution could be correlated to their oxidative and nitrosative capabilities, which may cause toxicity in eukaryotic cells. To investigate the scavenging ability of blood on reactive antimicrobial species during infusion experiments, the antimicrobial effect of plasma-treated NAC solution was tested on Escherichia coli ATCC 25922 in the presence of no blood or 1\%, 25\%, and 50\% of human blood, (obtained from the collaborative clinical laboratory). A single colony of E. coli was collected from a trypticase soy agar (TSA) plate (Thermo Fisher Scientific, Waltham, MA, USA), transferred into $10 \mathrm{~mL}$ of trypticase soy broth (TSB) (Thermo Fisher), and incubated in a shaker incubator at $37^{\circ} \mathrm{C}$ (at 120 revolutions per minute; rpm for $24 \mathrm{~h}$ ). The absorbance of grown E. coli culture was measured at $600 \mathrm{~nm}$ to arrange the absorbance to 0.2 which corresponds to about $10^{8}$ colony forming units (CFU)/mL of bacteria. Then the bacterial suspension was diluted to $10^{7} \mathrm{CFU} / \mathrm{mL}$ in $1 \mathrm{X}$ sterile PBS to use in antimicrobial (colony counting) assay experiments. Plasma-treated NAC was mixed with blood to have $1 \%$, $25 \%$, and $50 \%$ blood content. Plasma-treated NAC solutions with different blood content, were mixed with $10^{7} \mathrm{CFU} / \mathrm{mL}$ of E. coli separately and held together for $15 \mathrm{~min}$ at $37^{\circ} \mathrm{C}$. Thereafter samples were diluted in PBS and plated on TSA plates to incubate at $37^{\circ} \mathrm{C}$ for $24 \mathrm{~h}$. The next day, the colonies on TSA plates were counted; and plates with no growth were further incubated for another $24 \mathrm{~h}$ before noting as negative for growth. Antimicrobial assays were carried out three times in triplicate. Plasma-treated NAC with no blood content and plasma-untreated NAC were included as positive control and negative control groups, respectively. The plasma-treated NAC samples with different blood content were examined macroscopically and microscopically.

\subsection{Plasma-Treated NAC Solution and IV Catheter}

Plasma-treated NAC solutions (treated for 1,2, and $3 \mathrm{~min}$ ) or untreated solution was used for testing the patency of the catheter. An 18-G, closed iv catheter with a single port set (Becton-Dickinson and Company, Franklin Lakes, NJ, USA) was first flushed with normal saline, and then filled gently with either plasma-treated or untreated NAC solution, and clamp locked at both ends. The syringe port cap was replaced tightly. The catheter was checked for patency and damage every day for three consecutive days, with fresh NAC solutions refills, and observations noted. At the end of three days of NAC solution treatments, the catheter and port area were dissected, and macroscopic and microscopic (low power) observations made. A normal saline filled catheter was used as internal (negative) control.

\subsection{Administration of Plasma-Treated NAC Solution in Rats}

Figure 1 depicts an experimental schema. Different doses of 3-min plasma-treated NAC solution were administered to 3-month old female Sprague Dawley rats whose weight ranged 250-300 g. Rat infusion experiments were carried out in accordance with the Guide for the Care and Use of Laboratory Animals. The experimental procedure (Protocol \# 19512) was approved by the Institutional Animal Care and Use Committee (IACUC), Drexel University. Sprague Dawley rats were purchased from Harlan Laboratories (Indianapolis, IN, USA). In brief, rats were acclimatized for 5 days after their arrival in the animal laboratory. A total of 25 rats were randomly divided into five groups to have 5 rats in each. In the first group, no injection was performed; in the second group, $1 \mathrm{~mL}$ once a day untreated NAC solution was administered for 3 days; in the third group, $1 \mathrm{~mL}$ once a day 3-min plasma-treated NAC was administered for 3 days; in the fourth group, $1 \mathrm{~mL}$ three times a day of 3-min plasma-treated NAC solution was administered for one day; and in the fifth group, $1 \mathrm{~mL}$ three times a day 3-min plasma-treated NAC solution was administered for three days. Administration of plasma-treated NAC solutions was performed gently using a 20-gauge intravenous catheter via the tail vein. Prior to catheter placement, rats were anesthetized with 3\% isoflurane inhalation. Rats were closely observed for 1 week after completion of final injections. After 1 week of observation rats were euthanized with 
overdose sodium pentobarbital and phenytoin solution injection. Liver, kidneys, heart, lungs, tail vein portion, skin samples were collected from euthanized rats and sent to ANTECH Diagnostics (Lake Success, NY, USA) for histopathological evaluation. Also, blood samples were collected from the heart before scarifying.

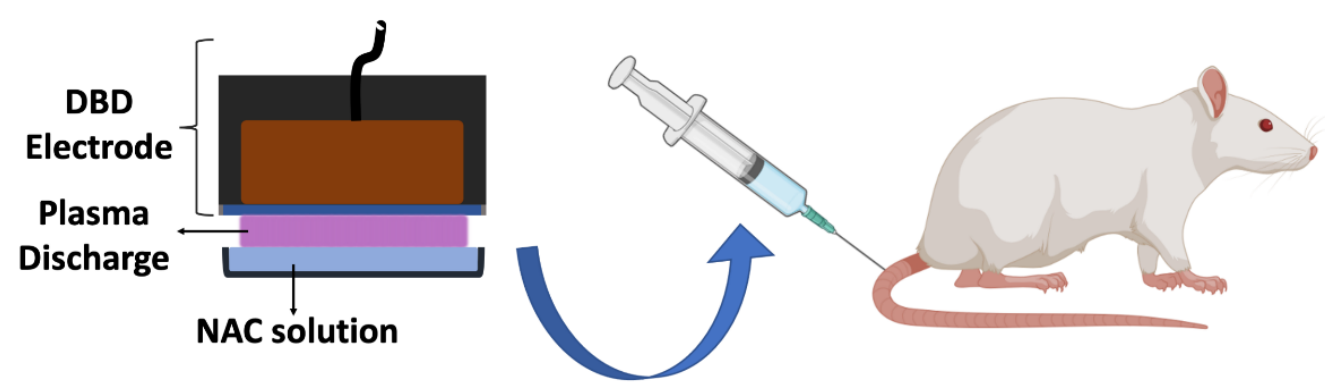

Figure 1. Experimental setup for treatment of NAC solution. After plasma treatment, NAC solution was injected into rats via tail vein.

\subsection{Statistical Analysis}

SPSS 13.0 was used for statistical analysis. All experiments were repeated at least three times in triplicate, unless otherwise stated. The data was represented as mean \pm standard deviation. Student $t$-test and one-way analysis of variance (ANOVA) were used for pair comparisons and multiple comparisons, respectively. Statistical significance was determined based on a $p$-value $(<0.05)$.

\section{Results}

\subsection{Cytotoxicity of Plasma-Treated NAC Solution}

Figure 2 shows the viability of EA.hy 926 human endothelial cells after 24 and $48 \mathrm{~h}$ of recovery period when they are exposed to 1,2, and 3-min plasma-treated NAC solutions for $5,10,15$, and $30 \mathrm{~min}$.

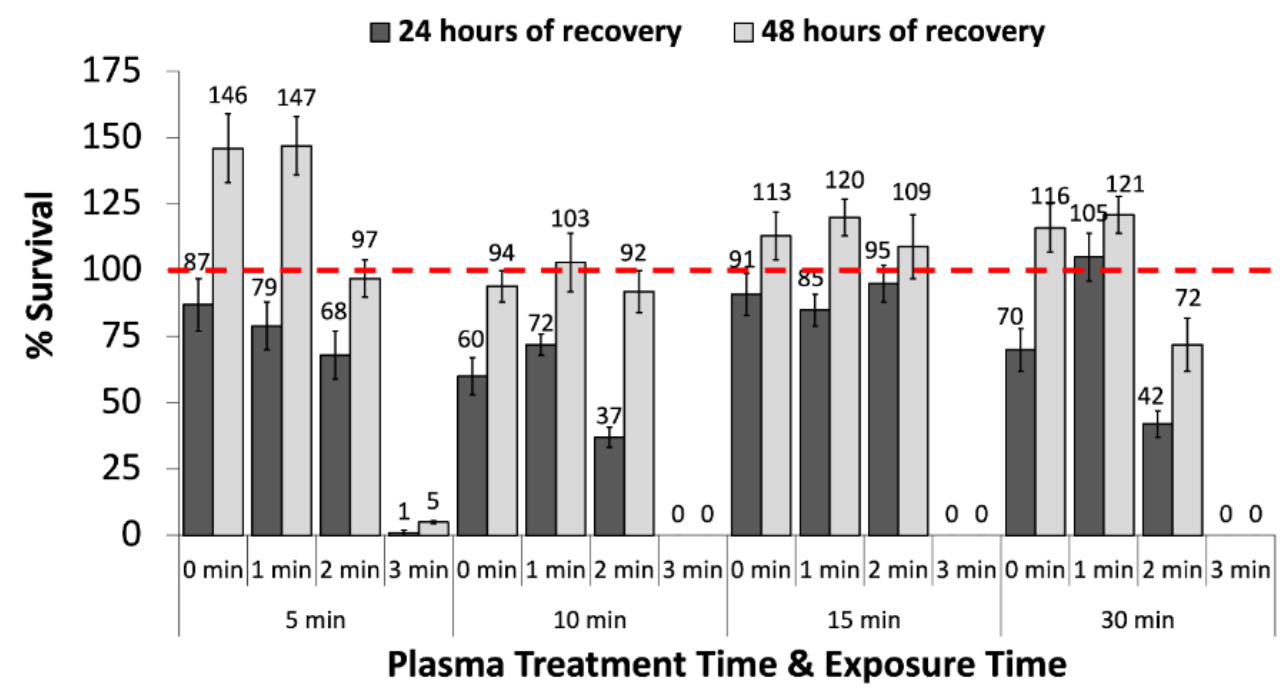

Figure 2. Cytotoxicity of plasma-treated NAC solution. Viabilities of EA.hy 926 human endothelial cells after 24 and 48 h of recovery period when exposed to 1, 2, and 3-min plasma-treated NAC solutions for 5, 10, 15, and $30 \mathrm{~min}$. The red dashed line represents a relative viability for the cells to $100 \%$ that were exposed to DMEM.

The dashed red line represents projected $100 \%$ viability, obtained from the cells that are only exposed to DMEM (nutrition media). The viability of cells that were exposed to $4 \%$ chlorhexidine was determined as 0 (zero; all cells are non-viable) and hence could 
not be graphed as bar (positive control of cytotoxicity). As demonstrated in the Figure 2, viability of the cells that were exposed to 3-min plasma-treated NAC solution for $5 \mathrm{~min}$ were $1 \%$ and $5 \%$ for 24 and $48 \mathrm{~h}$ of recovery, respectively, and the difference between them was insignificant, $(p>0.05)$. The cells that were exposed to 3-min plasma-treated NAC solution for 10,15, and 30 min lost their viability completely after 24 and $48 \mathrm{~h}$ of the recovery period. Viabilities of cells that were exposed to 1 and 2-min plasma-treated NAC solution were significantly higher after $48 \mathrm{~h}$ of recovery period compared to that of after $24 \mathrm{~h}$ recovery period for all exposure times $(p<0.05)$. Viability of the cells that were exposed to 1-min plasma-treated NAC solution for $5 \mathrm{~min}$ and cells that were exposed to 2-min plasma-treated NAC solution for 30 min were $147 \%$ and $72 \%$, respectively $(p<0.05)$. Except for those groups, viabilities of cells that were exposed to 1 and 2-min plasma-treated NAC solution are comparable to the viability of the (negative) control group (cells exposed to DMEM, shown as red dashed line) and are around $100 \%$ after $48 \mathrm{~h}$ of recovery period.

\subsection{Effect of Blood on Antimicrobial Efficacy of Plasma-Treated NAC Solution}

Consistent with our previous findings [18], 3-min plasma-treated NAC solution led 7-log inactivation of E. coli when exposed for $15 \mathrm{~min}$ as shown in Figure $3(p<0.05)$. Similarly, 7-log inactivation of E. coli was observed as well when bacteria were exposed to 3-min plasma-treated NAC solution in the presence of $1 \%$ blood $(p<0.05)$. However, the antimicrobial effect of 3-min plasma-treated NAC solution was completely lost in the presence of $25 \%$ and $50 \%$ blood (Figure 3 ).

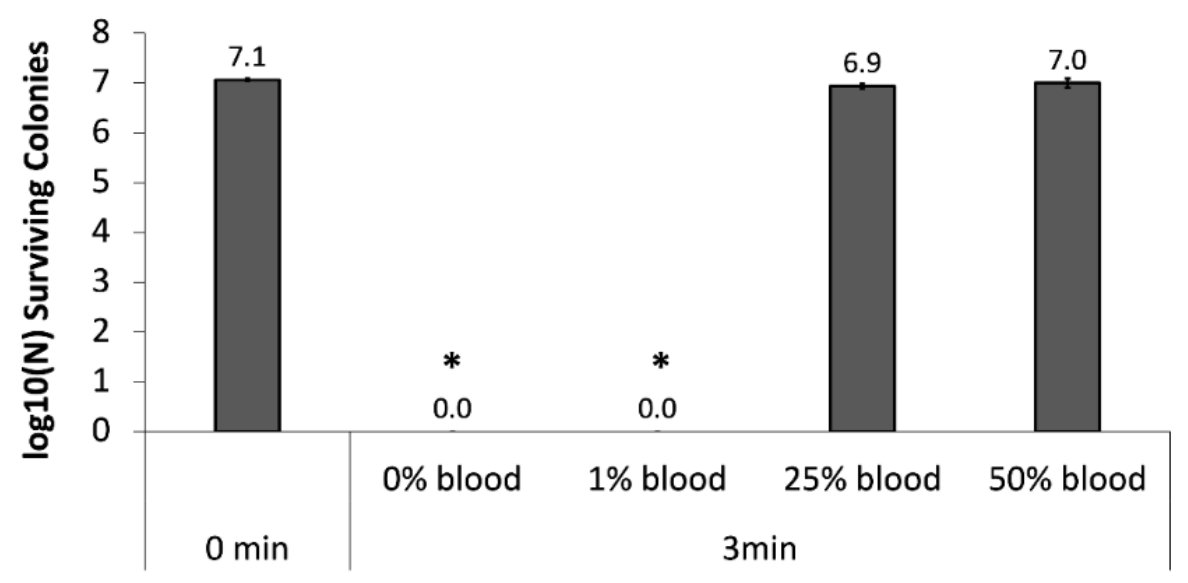

Plasma Treatment Time and Blood Content

Figure 3. Effect of $1 \%, 25 \%$, and $50 \%$ of blood content on the antimicrobial efficacy of 3-min plasmatreated NAC. ${ }^{*}$ denotes $p$-vaue $<0.05$.

Macroscopic observation of blood and 3-min plasma-treated NAC solution did not reveal any abnormal appearance (Figure $4 \mathrm{~A}$ ). The colors of the solutions after mixing with blood were found to be dependent on the percentage of blood. Microscopic observation of 3min plasma-treated NAC solution in the presence of $1 \%$ blood revealed normal morphology of erythrocytes (Figure 4B). As shown in Figure 4C, when the blood concentration was raised to $25 \%$ in 3-min plasma-treated NAC solution, irregular aggregation of erythrocytes was observed. For the samples with $50 \%$ blood, aggregation of erythrocytes was observed with evident rouleaux formation as exemplified with white arrows (Figure 4D).

\subsection{Plasma-Treated NAC Solution and IV Catheter Patency}

Throughout the experiments, none of the catheter lumens were found damaged, and catheter patency was found intact. During observations, the dissected catheter and port area were found smooth and undamaged like that of the untreated (negative control) catheter (data not shown). 


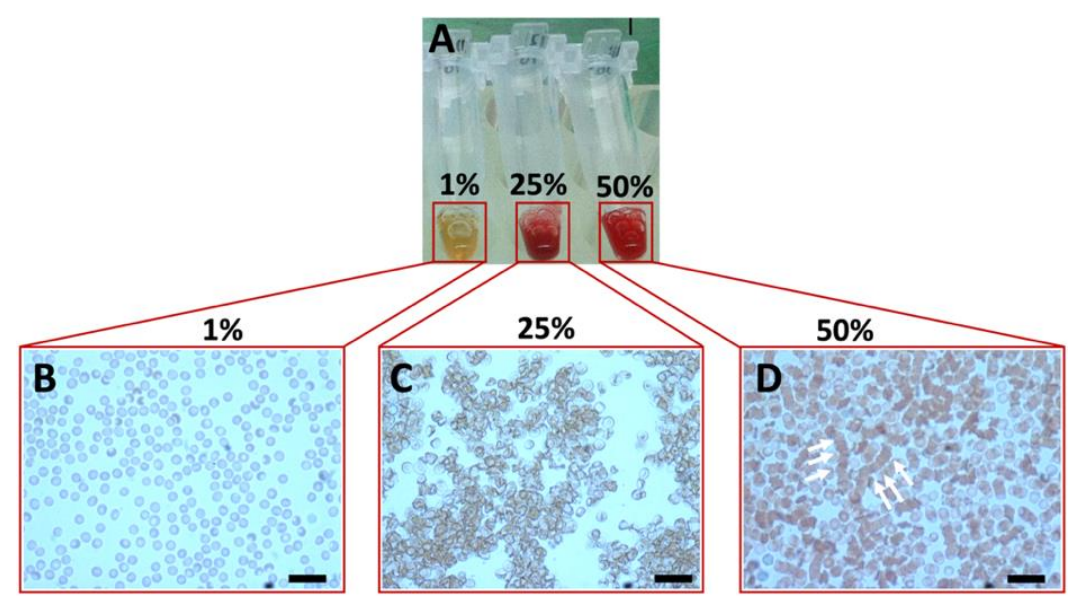

Figure 4. Macroscopic observation of a mixture containing 3-min plasma-treated NAC and 1\%, $25 \%$, and $50 \%$ of blood (A). The representative microscopic images of blood cells from the samples containing 3-min plasma-treated NAC solutions with $1 \%$ (B), $25 \%$ (C), and $50 \%$ (D) of blood. Scale bars represent $20 \mu \mathrm{m}$. White arrows indicate rouleaux formation.

\subsection{Systemic Toxicity of Plasma-Treated NAC Solution in Rats}

All animals were found healthy, and weight loss was not observed in any of the rats throughout one week of observation period after completion of infusions. As described under material and methods, after administration of 3-min plasma-treated NAC solution, the macroscopic and histopathological examinations were performed on liver, kidneys, heart, lungs, skin, and tail vein tissue samples. Table 1 narrates the findings of vital organ examination. The parenchymal organs (liver, lung, heart, and kidney) were having no abnormal lesions of any kind (Table 1$)$.

Table 1. The macroscopic and microscopic (histopathological) observation of vital organs on acute systemic toxicity (ultra-high doses) of day 3 study rats $(1 \mathrm{~mL} \times$ t.i.d. $\times 3$ days $)$.

\begin{tabular}{ll}
\hline System/Organ & Findings: Gross and Microscopic, Biochemical \\
\hline Liver & $\begin{array}{l}\text { Normal architecture; no hepatocellular degeneration/necrosis, } \\
\text { inflammation, or hepatocellular vacuolation; no abnormal lesions. }\end{array}$ \\
\hline Kidneys & $\begin{array}{l}\text { Most blood vessels, glomeruli, and renal tubules appear within } \\
\text { limits. Mild congestion in cortex. }\end{array}$ \\
\hline Heart & No structural changes, no abnormal lesions. \\
\hline Lungs & $\begin{array}{l}\text { Normal architecture; mild microvascular congestion; mild } \\
\text { cellular infiltration. }\end{array}$ \\
\hline Tail vein portion & Normal appearance of tissues; fibrotic change at site of injection. \\
\hline Skin & $\begin{array}{l}\text { Epidermal and dermal cells and micro-vessels appear within } \\
\text { normal limits; mild lymphocytic, macrophagic, and scattered } \\
\text { neutrophilic infiltration. }\end{array}$ \\
\hline Blood cells & WBC slightly lowered; other cells in normal limit. \\
\hline Serum proteins & Within normal limits. \\
\hline Serum enzymes & Within normal limits. \\
\hline Others; serum electrolytes & Within normal limits; creatinine normal. \\
\hline
\end{tabular}

Special attention was directed to the kidneys and liver, which are the concentrating, metabolizing, and detoxicating organs and are most likely to be affected by toxic substances. Neither kidney nor liver in any of the rats exhibited any abnormal lesions during the microscopic examination that can be correlated to toxicity. There was no hepatocellular degeneration/necrosis, inflammation, or hepatocellular vacuolation. Mild to moderate 
microvascular congestion was a common feature shared by the kidney, liver, and lung. It was either normal physiological congestion or perhaps artifactual congestion that is associated with terminal agonal cardiovascular collapse. Mild to moderate peribronchiolar lymphoid hyperplasia was observed in most of the rats (independent of experimental group), which is a very common observation even in normal, healthy, non-experimental rats, representing a cellular response to inhalant antigens and/or irritants, and also most likely unrelated to the experimental protocol. This was a pulmonary arteriolar adaptation to vascular hypertension, which may be spontaneous or induced but generally not a sign of toxicity. There were instances of mild to moderate tail vein thrombosis with generally mild non-necrotizing vasculitis and peri-vasculitis in a few instances also mild perivascular fibrosis (independent of experimental group). However, the vascular lesions were not sufficient to have caused regional insufficiency (infarction). The tail veins distal to the injection site were normal for all experimental groups. Overall, the histopathological examination did not indicate any toxicity which might have been caused by 3-min plasmatreated NAC solution on liver, kidney, heart, lung, skin, and tail vein tissue samples. Even though there are some variations, blood tests remain reasonably within or close to normal limits and do not represent any specific signs of toxicity. Table 2 summarizes the results of the blood test compared to the reference values.

Table 2. Some important blood parameter observation on acute systemic toxicity against high, and ultrahigh doses of 3-min plasma-treated NAC solution. The variations in blood parameters are compared to normal reference values of rats. E, slightly elevated as compared to reference value; L, relatively lowered as compared to reference value; AST, aspartate transaminase; ALT, alanine transaminase; ALP, alkaline phosphatase; WBC, white blood cells. All other values are within the normal range. A q.d., once a day; t.i.d., three times a day, for indicated days.

\begin{tabular}{|c|c|c|c|c|c|}
\hline & No Injection & Untreated NAC & $1 \mathrm{~mL}$ q.d. $\times 3$ days & $1 \mathrm{~mL}$ t.i.d. $\times 1$ day & $1 \mathrm{~mL}$ t.i.d. $\times 3$ days \\
\hline AST & E & E & E & $\mathrm{E}$ & $\mathrm{E}$ \\
\hline ALT & E & $\mathrm{E}$ & & $\mathrm{E}$ & \\
\hline ALP & E & $\mathrm{E}$ & & $\mathrm{E}$ & \\
\hline Glucose & $\mathrm{E}$ & & $\mathrm{E}$ & $\mathrm{E}$ & $\mathrm{E}$ \\
\hline Creatinine & $\mathrm{L}$ & & & & \\
\hline $\mathrm{K}^{+}$ & & $\mathrm{E}$ & & $\mathrm{E}$ & \\
\hline WBC & & $\mathrm{L}$ & & $\mathrm{L}$ & $\mathrm{L}$ \\
\hline $\mathrm{PO}_{4}^{-3}$ & & & & $\mathrm{E}$ & $\mathrm{E}$ \\
\hline $\mathrm{Na}^{+}$ & & & & & $\mathrm{L}$ \\
\hline Globulin & & & & $\mathrm{L}$ & \\
\hline
\end{tabular}

Both Tables 1 and 2 demonstrate, none of these variations can be correlated to any specific experimental group. Furthermore, these variations are not linked to any toxicity.

\section{Discussion}

Plasma-treated liquids are promising antimicrobial solutions to surface-associated pathogenic contaminants in their planktonic as well as biofilm forms, and they exhibit broad-spectrum activity against MDR pathogens [18,23]. Among them, plasma-treated NAC solution was observed as a strong and stable solution which retained its broadspectrum antimicrobial property over two years [18], making it eligible for storage and use (whenever required) during an extended period of time. Their strong antimicrobial effect is usually attributed to ROS and RNS that are generated in it by plasma treatment. These ROS and RNS either diffuse into and/or chemically modify and stabilize in treated liquid and make it a potent antimicrobial [20]. Both, CRBSI and CLABSI are major surface contamination-associated hospital-acquired infections, wherein lock solutions are used to guard the intravenous (IV) ports and keep the patency of iv catheters. The main concern about almost all lock solutions that are commercially available or clinically used is either their toxicity (due to their leakage in systemic circulation) or they promote drug resistance among other nosocomial, or do not have sufficient antimicrobial properties and merely 
keeping catheter patency [4]. An ideal antimicrobial catheter lock solution is expected to have features such as broad-spectrum antimicrobial and antibiofilm activity against various MDR pathogens, extended stability, compatibility with catheters that are fabricated with various materials, minimal possibility of resistance development, cost effectiveness, and minimal risk for toxicity [2,11]. Many of these properties are reported for various plasma-treated liquids by our laboratory or from elsewhere $[18,20]$. The studies have shown no sign of resistance development in consequence of repetitive cold atmospheric plasma treatments, albeit they were direct plasma treatment instead of application of plasma-treated liquids [24]. The present study is focused on the evaluation of the cellular and systemic toxicity of plasma-treated NAC solution, and the blood's scavenging effect on this antimicrobial plasma solution when it gets diluted.

Traditionally, NAC is an antioxidant agent and used as a scavenger of ROS in various cold atmospheric plasma studies [25]. However, when NAC is treated with DBD cold atmospheric plasma in air, its thiol group is replaced by NO that turns the molecule to S-nitroso $\mathrm{N}$-acetylcysteine (SNAC). SNAC acts as a nitric oxide donor and presents strong oxidative and nitrosative properties that make it a strong antimicrobial [20]. Strong antimicrobial effect of plasma-treated NAC solution was shown on the biofilms of several pathogens including Pseudomonas aeruginosa, Staphylococcus aureus, Candida glabrata, Staphylococcus epidermidis that were grown on polyurethane catheter slices, and they were reported as common pathogens responsible for CLABSI and CRBSI [1,18].

Selectivity of plasma treatment in between bacterial and eukaryotic cells has been reported [24,26], and bacteria are reportedly found more susceptible to inactivation than eukaryotic cells. Upon 15 min exposure of EA.hy926 human endothelial cells to 2-min plasma-treated NAC solution, the recovery period of $24 \mathrm{~h}$ and $48 \mathrm{~h}$ allowed cell numbers to restore to $95 \%$ and $100 \%$ respectively, indicates the cellular tolerance; but 3-min plasmatreated NAC solutions found toxic to cells, probably generating large of reactive species, beyond their tolerance, and could not be restored. These results also indicate the strong oxidative and nitrosative power of the 3-min plasma-treated NAC solution. It is important to emphasize that cytotoxicity experiments were carried out in a 96-well plate that had $\sim 3 \times 10^{4}$ cells when wells are confluent. Therefore, 2-min plasma-treated NAC solution was also exposed to $5 \times 10^{4}, 10^{5}$, and $10^{6} \mathrm{CFU} / \mathrm{mL}$ E. coli for $15 \mathrm{~min}$ to evaluate whether its antimicrobial activity is enough to inactivate given bacterial concentrations; and the findings confirm total inactivation (data not shown, as these findings are already on public domain). This also in part supports the selectivity of plasma-treated NAC solution in between prokaryotic cells and eukaryotic cells. The selectivity of plasma on bacterial and eukaryotic cells was attributed to several factors such, as the presence of membrane-bound organelles, more developed antioxidant systems, DNA repair mechanism/machinery, and resistance mechanism to external stress in eukaryotic cells along with size difference in between bacterial and eukaryotic cells [27]. In a real scenario, the cells inside the body are well organized in the form of tissues, and it is reasonable to expect a collective robust resistance response to any chemical or antimicrobial agents versus in vitro monolayered cells.

A demonstration of complete loss of antimicrobial property of plasma-treated NAC solution by a mere presence of less than $25 \%$ of blood is important (Figure 3), as it suggests that even if there would be a leakage of minute amounts of this solution to systemic circulation, the solution's antimicrobial property would be neutralized, rendering it nonreactive. This scavenging or quenching effect of blood on antimicrobial solution is also an indicator of the loss of the oxidative (ROS) and nitrosative (RNS) effect of plasmatreated NAC solution. This might be due to the large amount of proteins and other organic substances present in the blood, and a systematic study of individual components would be interesting to note in the future. A loss of antimicrobial effect of plasma-treated liquid in the presence of proteins is reported [28]. Thus, the loss of antimicrobial effect of plasma-treated NAC in the presence of a minute amount of blood would not be a concern regarding the use of this solution as an antimicrobial catheter lock solution. In other words, the effective flushing of the catheter lumen with normal saline before the administration of 
the lock solution, would wash out the blood and preserve plasma-treated NAC solution's antimicrobial potency as well. This will also enable the prevention of biofilm formation, and eradication of the biofilm-embedded pathogen if any. Plasma-treated solutions are known to inactivate biofilm-embedded pathogens and inhibit biofilm formation [18]. During in vitro catheter patency testing, the macroscopic and low power microscopic observations did not reveal any damage to internal luminal surfaces and port areas. The findings suggest that all plasma-treated NAC solutions for various treatment times are completable, keeping patency, and catheter-friendly.

A macroscopic investigation of blood exposed to plasma-treated-NAC solutions was performed and did not reveal any abnormal feature. The consistency of all blood and plasma-treated NAC-solution mixtures was similar in appearance and showed no sign of hemolysis upon close observation by the naked eye. Bubbles visible in Figure 4A were due to pipetting for homogenization of samples. The color difference observed among the tubes containing $1 \%, 25 \%$, and $50 \%$ of blood can be attributed to the dilution of blood in plasmatreated NAC solution. On the other hand, microscopic observation elicited differences in blood cell morphology. The aggregation of erythrocytes and rouleaux formation, especially in tubes containing $50 \%$ blood was observed. Direct plasma treatment generating ROS or RNS has shown to accelerate blood coagulation via aggregation of platelets and subsequent clumping of erythrocytes [29], and a similar effect could be expected by plasma-treated liquids (especially, when it contains high concentrations of ROS/RNS; as observed in 3-min plasma-treated NAC solution). Platelets are remarkably smaller than erythrocytes and seen as blue-purple refractile bodies when stained (especially when images are zoomed in). A clumping of erythrocytes seen in Figure 4C could be partly attributed to platelet activation by plasma-treated NAC solution however, this effect is believed to be minimal, as no sign of coagulation was not observed macroscopically. While the rouleaux formation in the presence of $50 \%$ of blood could be attributed to lack of flow, and its link to plasma-treated NAC solution needs further research. Aggregations of blood cells of various degrees occur most of the time in intravenous catheters. The efficient flushing of the catheter would wash off such blood stagnated from the lumen, and prevent the coagulation when plasma treat NAC solution is used as lock solution.

Although various studies have shown dose-dependent toxicity as the localized effect of non-thermal plasma on different tissues described by other investigators, studies regarding systemic administration of cold plasma-treated liquids and their systemic toxicities are limited $[30,31]$ and deserve further investigations. To the best of our knowledge, this study is the first that evaluates the administration of a plasma-treated liquid to the systemic circulation and looks at its systemic toxicity.

The peritoneum is a route for the administration of various pharmacological compounds since the compound is absorbed by lymphatic vessels and capillaries and enters systemic circulation much faster [32]. Previously, our colleagues have performed direct plasma treatment on the peritoneum of mice to investigate the effect of cold atmospheric plasma on the prevention of peritoneal adhesions and did not observe any sign of toxicity, but it was a short duration of treatment and a comparably low dose of plasma [33]. It is noteworthy that the average blood volume of female Sprague Dawley rats is approximately $7.8 \mathrm{~mL} / 100 \mathrm{~g}$ that corresponds to an average blood volume of $21 \mathrm{~mL}$ for the animals used in the present study [34]. The maximum administrated volume of the 3-min plasma-treated NAC in a day was $3 \mathrm{~mL}$ that makes about one seventh of the total blood volume of the rat. When compared to the blood volume of a healthy human, which is about $5 \mathrm{~L}$, this volume corresponds to about $700 \mathrm{~mL}$ of plasma-treated liquid administration which is significantly higher than the amount of the catheter lock solution that may leak in systemic circulation as the approximate volume of lock solution installation ranges between 1 to $5 \mathrm{~mL}$ [2]. We had intentionally selected ultra-high doses of proposed solutions to observe the upper end of toxicities, determine tolerance or safety margin. The findings on vital organs (Table 1) and blood analysis (Table 2) explain systemic tolerance. The previously defined experiments of the effect of the presence of proteins leading to loss of antimicrobial effect of plasma-treated 
liquids, indicates the scavenging of plasma generated reactive species [28]. Thus, the lack of any sign of systemic toxicity upon injection of 3-min plasma-treated NAC solution can be attributed to scavenging of plasma generated reactive species by high content of proteins in blood. Overall, when considering the relatively high doses of administrated 3-min plasmatreated NAC solution with no observed toxicity, the use of 3-min plasma-treated NAC solution as a catheter lock solution seems appropriate and safe. However, the present study investigates only short-term systemic toxicity of 3-min plasma-treated NAC solution, and the long-term evaluations would be interesting to follow. Through previously published studies the authors reported plasma-treated NAC solution's broad-spectrum antimicrobial and antibiofilm properties against clinically relevant Gram-positive and Gram-negative pathogens [18]. Therefore, in the present study, the authors selected a widely adopted model organism, E. coli to test the effect of plasma-treated NAC solution in the presence of blood.

\section{Conclusions}

Despite the well-known antimicrobial effects of atmospheric cold plasma and the atmospheric cold plasma-treated liquids, their applicability in vivo especially for control and prevention of hospital acquired infections is still elusive. In the present study, the useability of plasma-treated NAC solution was evaluated as a catheter lock solution along with its possible cellular and systemic responses in vitro and in vivo. The findings suggest that the cytotoxic effects of plasma-treated NAC solution are dependent on plasmatreatment time, surface/biomaterial exposure (contact) time, and the number of cells. Strong antimicrobial effect with no cytotoxicity or systemic toxicity of plasma-treated NAC solution is achievable. Based on the findings together, we conclude that the atmospheric cold plasma-treated NAC solution can be considered as a safe antimicrobial lock solution for the control and prevention of catheter-related bloodstream infections.

Author Contributions: Conceptualization, S.G.J., A.D.B. and U.K.E.; methodology, U.K.E., A.D.Y. and K.W.; validation, S.G.J., U.K.E. and A.D.Y.; formal analysis, S.G.J. and U.K.E.; investigation, S.G.J. and U.K.E.; resources, S.G.J. and A.D.B.; data curation, S.G.J. and U.K.E.; writing—original draft preparation, U.K.E. and S.G.J.; writing-review and editing, U.K.E., A.D.Y., S.G.J. and A.D.B.; visualization, U.K.E.; supervision, S.G.J. and A.D.B.; project administration, S.G.J. and A.D.B.; funding acquisition, S.G.J. and A.D.B. All authors have read and agreed to the published version of the manuscript.

Funding: This research was supported through the intramural funding of the research division of Department of Surgery of Drexel University.

Institutional Review Board Statement: The experimental procedure (Protocol \# 19512) was approved by the Institutional Animal Care and Use Committee (IACUC), Drexel University. No human subjects were involved in this study. The Drexel University clinical laboratory blood sample used was waived and unidentified. Thus, ethical review and approval was waived for this study.

Informed Consent Statement: Not applicable.

Data Availability Statement: No additional data relevant to this research at this point other than presented in this manuscript.

Acknowledgments: The authors thank the department of surgery and the department of laboratory of animal research of Drexel University.

Conflicts of Interest: The authors declare no conflict of interest.

\section{References}

1. Ruiz-Giardin, J.M.; Chamorro, I.O.; Ríos, L.V.; Aroca, J.J.; Arata, M.I.G.; López, J.V.S.; Santillán, M.G. Blood stream infections associated with central and peripheral venous catheters. BMC Infect. Dis. 2019, 19, 1-9. [CrossRef]

2. Rupp, M.E.; Karnatak, R. Intravascular catheter-related bloodstream infections. Infect. Dis. Clin. 2018, 32, 765-787. [CrossRef] [PubMed]

3. Bell, T.; O'Grady, N.P. Prevention of central line-associated bloodstream infections. Infect. Dis. Clin. 2017, 31, 551-559. [CrossRef] 
4. Labriola, L. Antibiotic locks for the treatment of catheter-related blood stream infection: Still more hope than data. In Proceedings of the Seminars in Dialysis; Wiley Online Library: Hoboken, NJ, USA, 2019; Volume 32, pp. 402-405.

5. $\quad$ O'grady, N.P.; Alexander, M.; Burns, L.A.; Dellinger, E.P.; Garland, J.; Heard, S.O.; Lipsett, P.A.; Masur, H.; Mermel, L.A.; Pearson, M.L. Guidelines for the prevention of intravascular catheter-related infections. Clin. Infect. Dis. 2011, 52, e162-e193. [CrossRef] [PubMed]

6. Mermel, L.A. What is the evidence for intraluminal colonization of hemodialysis catheters? Kidney Int. 2014, 86, 28-33. [PubMed]

7. Labriola, L.; Pochet, J.-M. Any use for alternative lock solutions in the prevention of catheter-related blood stream infections? J. Vasc. Access 2017, 18, S34-S38. [CrossRef]

8. Zacharioudakis, I.M.; Zervou, F.N.; Arvanitis, M.; Ziakas, P.D.; Mermel, L.A.; Mylonakis, E. Antimicrobial lock solutions as a method to prevent central line-associated bloodstream infections: A meta-analysis of randomized controlled trials. Clin. Infect. Dis. 2014, 59, 1741-1749. [CrossRef] [PubMed]

9. Umscheid, C.A.; Mitchell, M.D.; Doshi, J.A.; Agarwal, R.; Williams, K.; Brennan, P.J. Estimating the proportion of healthcareassociated infections that are reasonably preventable and the related mortality and costs. Infect. Control Hosp. Epidemiol. 2011, 32, 101-114. [CrossRef] [PubMed]

10. Bustos, C.; Aguinaga, A.; Carmona-Torre, F.; Del Pozo, J.L. Long-term catheterization: Current approaches in the diagnosis and treatment of port-related infections. Infect. Drug Resist. 2014, 7, 25. [PubMed]

11. Justo, J.A.; Bookstaver, P.B. Antibiotic lock therapy: Review of technique and logistical challenges. Infect. Drug Resist. 2014, 7, 343. [PubMed]

12. Centers for Disease Control and Prevention. Vital signs: Central line-associated blood stream infections-United States, 2001, 2008, and 2009. Ann. Emerg. Med. 2011, 58, 447-450.

13. Goossens, G.A. Flushing and locking of venous catheters: Available evidence and evidence deficit. Nurs. Res. Pract. 2015, 2015, 985686. [PubMed]

14. Messing, B.; Peitra-Cohen, S.; Debure, A.; Beliah, M.; Bernier, J. Antibiotic-lock technique: A new approach to optimal therapy for catheter-related sepsis in home-parenteral nutrition patients. J. Parenter. Enter. Nutr. 1988, 12, 185-189. [CrossRef] [PubMed]

15. Bookstaver, P.B.; Rokas, K.E.E.; Norris, L.B.; Edwards, J.M.; Sherertz, R.J. Stability and compatibility of antimicrobial lock solutions. Am. J. Health Pharm. 2013, 70, 2185-2198. [CrossRef] [PubMed]

16. Raad, I.; Bodey, G.P., Sr. Novel antimicrobial catheter lock solution: A new direction in which chelators replace heparin. Crit. Care Med. 2011, 39, 875-876. [CrossRef]

17. Bernhardt, T.; Semmler, M.L.; Schäfer, M.; Bekeschus, S.; Emmert, S.; Boeckmann, L. Plasma medicine: Applications of cold atmospheric pressure plasma in dermatology. Oxid. Med. Cell. Longev. 2019, 2019, 3873928. [CrossRef]

18. Ercan, U.K.; Wang, H.; Ji, H.; Fridman, G.; Brooks, A.D.; Joshi, S.G. Nonequilibrium plasma-activated antimicrobial solutions are broad-spectrum and retain their efficacies for extended period of time. Plasma Process. Polym. 2013, 10, 544-555. [CrossRef]

19. Joshi, S.G.; Paff, M.; Friedman, G.; Fridman, G.; Fridman, A.; Brooks, A.D. Control of methicillin-resistant Staphylococcus aureus in planktonic form and biofilms: A biocidal efficacy study of nonthermal dielectric-barrier discharge plasma. Am. J. Infect. Control 2010, 38, 293-301. [CrossRef]

20. Ercan, U.K.; Smith, J.; Ji, H.-F.; Brooks, A.D.; Joshi, S.G. Chemical Changes in Nonthermal Plasma-Treated N-Acetylcysteine (NAC) Solution and Their Contribution to Bacterial Inactivation. Sci. Rep. 2016, 6, 20365. [CrossRef] [PubMed]

21. Sakiyama, Y.; Tomai, T.; Miyano, M.; Graves, D.B. Disinfection of E. coli by nonthermal microplasma electrolysis in normal saline solution. Appl. Phys. Lett. 2009, 94, 161501. [CrossRef]

22. Ercan, U.K.; Sen, B.; Brooks, A.D.; Joshi, S.G. Escherichia coli cellular responses to exposure to atmospheric-pressure dielectric barrier discharge plasma-treated N-acetylcysteine solution. J. Appl. Microbiol. 2018, 125, 383-397. [CrossRef]

23. Handorf, O.; Pauker, V.I.; Schnabel, U.; Weihe, T.; Freund, E.; Bekeschus, S.; Riedel, K.; Ehlbeck, J. Characterization of antimicrobial effects of plasma-treated water (PTW) produced by microwave-induced plasma (MidiPLexc) on Pseudomonas fluorescens biofilms. Appl. Sci. 2020, 10, 3118. [CrossRef]

24. Wende, K.; Landsberg, K.; Lindequist, U.; Weltmann, K.-D.; von Woedtke, T. Distinctive activity of a nonthermal atmosphericpressure plasma jet on eukaryotic and prokaryotic cells in a cocultivation approach of keratinocytes and microorganisms. IEEE Trans. Plasma Sci. 2010, 38, 2479-2485. [CrossRef]

25. Liu, Y.; Tan, S.; Zhang, H.; Kong, X.; Ding, L.; Shen, J.; Lan, Y.; Cheng, C.; Zhu, T.; Xia, W. Selective effects of non-thermal atmospheric plasma on triple-negative breast normal and carcinoma cells through different cell signaling pathways. Sci. Rep. 2017, 7, 1-12. [CrossRef] [PubMed]

26. Pai, K.K.; Singarapu, K.; Jacob, J.D.; Madihally, S. V Dose dependent selectivity and response of different types of mammalian cells to surface dielectric barrier discharge (SDBD) plasma. Plasma Process. Polym. 2015, 12, 666-677. [CrossRef]

27. Dobrynin, D.; Fridman, G.; Friedman, G.; Fridman, A. Physical and biological mechanisms of direct plasma interaction with living tissue. N. J. Phys. 2009, 11, 115020. [CrossRef]

28. Hahn, V.; Dikyol, C.; Altrock, B.; Schmidt, M.; Wende, K.; Ercan, U.K.; Weltmann, K.-D.; von Woedtke, T. Plasma-mediated inactivation of E. coli: Influence of protein on wet surface and in liquid medium. Plasma Process. Polym. 2019, 16, 1800164. [CrossRef]

29. Kalghatgi, S.U.; Fridman, G.; Cooper, M.; Nagaraj, G.; Peddinghaus, M.; Balasubramanian, M.; Vasilets, V.N.; Gutsol, A.F.; Fridman, A.; Friedman, G. Mechanism of blood coagulation by nonthermal atmospheric pressure dielectric barrier discharge plasma. IEEE Trans. Plasma Sci. 2007, 35, 1559-1566. [CrossRef]

30. Boehm, D.; Bourke, P. Safety implications of plasma-induced effects in living cells-a review of in vitro and in vivo findings. Biol. Chem. 2018, 400, 3-17. [CrossRef] [PubMed] 
31. Dobrynin, D.; Wu, A.; Kalghatgi, S.; Park, S.; Chernets, N.; Wasko, K.; Dumani, E.; Ownbey, R.; Joshi, S.G.; Sensenig, R. Live pig skin tissue and wound toxicity of cold plasma treatment. Plasma Med. 2011, 1, 93-108. [CrossRef]

32. Turner, P.V.; Brabb, T.; Pekow, C.; Vasbinder, M.A. Administration of substances to laboratory animals: Routes of administration and factors to consider. J. Am. Assoc. Lab. Anim. Sci. 2011, 50, 600-613.

33. Gökçelli, U.; Ercan, U.K.; İlhan, E.; Argon, A.; Çukur, E.; Üreyen, O. Prevention of Peritoneal Adhesions by Non-Thermal Dielectric Barrier Discharge Plasma Treatment on Mouse Model: A Proof of Concept Study. J. Investig. Surg. 2019, 33, 605-614. [CrossRef] [PubMed]

34. Probst, R.J.; Lim, J.M.; Bird, D.N.; Pole, G.L.; Sato, A.K.; Claybaugh, J.R. Gender differences in the blood volume of conscious Sprague-Dawley rats. J. Am. Assoc. Lab. Anim. Sci. 2006, 45, 49-52. [PubMed] 